\title{
Kinetics and Mechanism of Plasmon-Driven Dehalogenation Reaction of Brominated Purine Nucleobases on Ag and Au
}

\author{
Anushree Dutta, Robin Schürmann, Sergio Kogikoski, Jr., Niclas S. Mueller, Stephanie Reich, \\ and Ilko Bald*
}

Cite This: ACS Catal. 2021, 11, 8370-8381

Read Online

ACCESS | Lill Metrics \& More | 国 Article Recommendations ｜ st Supporting Information

ABSTRACT: Plasmon-driven photocatalysis is an emerging and promising application of noble metal nanoparticles (NPs). An understanding of the fundamental aspects of plasmon interaction with molecules and factors controlling their reaction rate in a heterogeneous system is of high importance. Therefore, the dehalogenation kinetics of 8-bromoguanine (BrGua) and 8bromoadenine (BrAde) on aggregated surfaces of silver $(\mathrm{Ag})$ and gold ( $\mathrm{Au}$ ) NPs have been studied to understand the reaction kinetics and the underlying reaction mechanism prevalent in heterogeneous reaction systems induced by plasmons monitored by surface enhanced Raman scattering (SERS). We conclude that the time-average constant concentration of hot electrons and the time scale of dissociation of transient negative ions (TNI) are crucial in defining the reaction rate law based on a proposed kinetic model. An overall higher reaction rate of dehalogenation is observed on $\mathrm{Ag}$ compared with $\mathrm{Au}$, which is

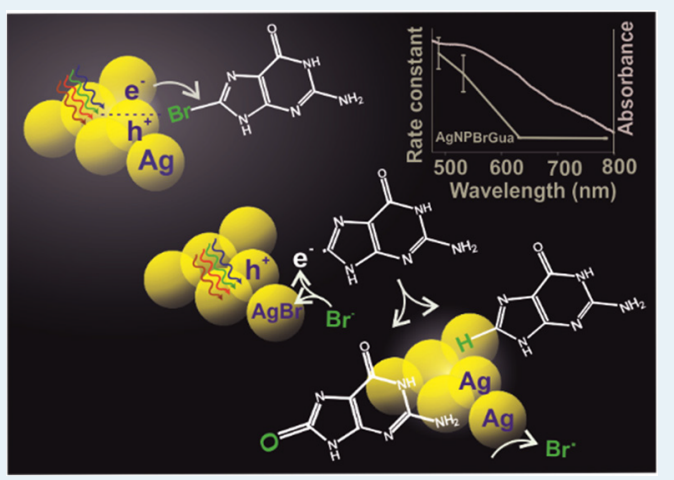
explained by the favorable hot-hole scavenging by the reaction product and the byproduct. We therefore arrive at the conclusion that insufficient hole deactivation could retard the reaction rate significantly, marking itself as rate-determining step for the overall reaction. The wavelength dependency of the reaction rate normalized to absorbed optical power indicates the nonthermal nature of the plasmon-driven reaction. The study therefore lays a general approach toward understanding the kinetics and reaction mechanism of a plasmon-driven reaction in a heterogeneous system, and furthermore, it leads to a better understanding of the reactivity of brominated purine derivatives on $\mathrm{Ag}$ and $\mathrm{Au}$, which could in the future be exploited, for example, in plasmon-assisted cancer therapy. KEYWORDS: hot-electrons, plasmon-driven catalysis, fractal kinetics, brominated purines, SERS, hole scavengers

\section{INTRODUCTION}

Visible-light-driven chemical reactions on the surface of plasmonic nanostructures form the basis of plasmon chemistry. That is to say, an incident photon of certain energy when impinged on the surface of a nanoparticle (NP) can trigger the collective oscillation of the free electron cloud which results in the generation of a localized surface plasmon (LSP). ${ }^{1-3}$ When the oscillating electron cloud dephases, electron-electron scattering on the NP surface results in the generation of nonequilibrium high-energy electrons for a short time scale that are referred to as "hot electrons". 4 "The hot electrons (charge carriers) can easily get transferred to an unoccupied orbital of a nearby chemisorbed or physisorbed reactant molecule to induce a chemical reaction within the system via transient electronic exchange. This is termed as a surface plasmon (SP)-induced reaction and is often studied by SERS. ${ }^{6,7}$

Several mechanistic models described by dissociative electron attachment (DEA), ${ }^{8}$ desorption induced by electronic transition (DIET), and desorption induced by multiple electronic transitions (DIMET), ${ }^{9,10}$ among others, have been put forward to study and understand the nature of some exemplary plasmon-induced reactions. For example, the mechanism of plasmon-induced dissociation reactions of $\mathrm{O}_{2}$ and $\mathrm{H}_{2}$ has been explained on the basis of the formation and relaxation of transient negative ions (TNIs) resulting in vibrational excitation of the neutral molecules. ${ }^{9,11,12}$ On a similar ground, an initial report from our group demonstrates the $\mathrm{C}-\mathrm{Br}$ bond cleavage in 8-bromoadenine (BrAde) adsorbed on aggregates of $\mathrm{Au}$ and $\mathrm{Ag}$ via hot electron transfer based on the DEA model. ${ }^{8}$ Along this line, an understanding over controlling and increasing the efficiency of the same plasmoninduced reaction was laid forward by Liu et al. ${ }^{13}$ using different applied potentials and laser light. In principle, a three-step mechanism based on DEA has been proposed which is generalized as follows: $:^{3,8,13}$

Received: April 23, 2021

Revised: June 7, 2021

Published: June 23, 2021 

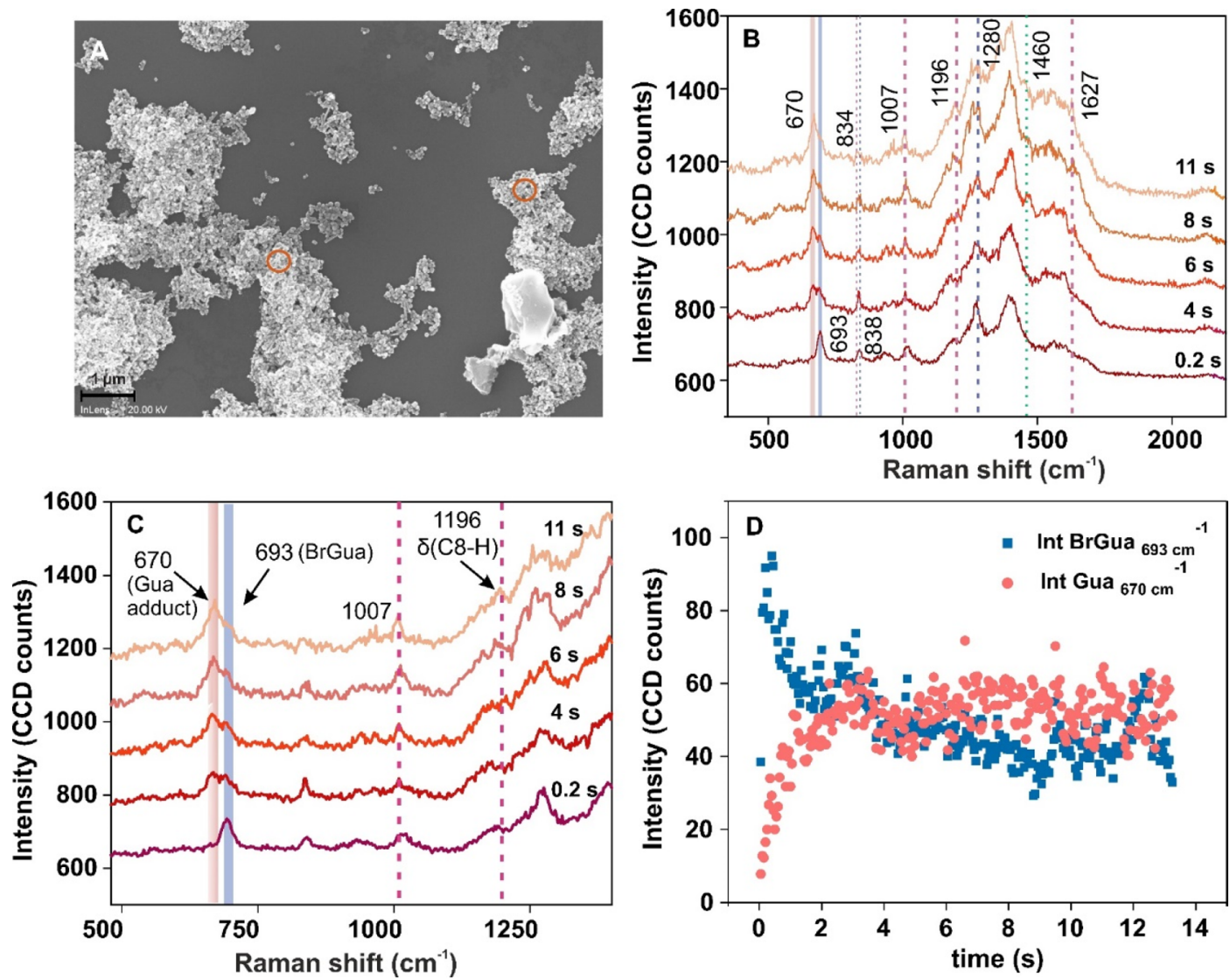

Figure 1. (A) Representative FESEM image of BrGua-coated aggregated AgNPs. (B) SERS spectra recorded during BrGua dissociation on aggregated AgNPs under CW illumination at different time intervals using a $532 \mathrm{~nm}$ laser source (laser power: $0.2 \mathrm{~mW}$; data acquisition time: 0.05 s; accumulation: 300 ) and marked bands in blue and pink correspond to the ring breathing mode of BrGua and Gua adduct, respectively, shown as (C) zoom-in view of the SERS spectra $\left(500-1300 \mathrm{~cm}^{-1}\right)$ recorded in panel A. (D) Plot showing change in SERS intensity of the ring-breathing mode of BrGua at $670 \mathrm{~cm}^{-1}$ against that of the Gua adduct at $693 \mathrm{~cm}^{-1}$ as a function of laser illumination time.

(i) The first step involves the generation of hot electrons upon laser illumination at a selected wavelength described by eq 1 .

$$
\mathrm{NP}+h v \underset{-k_{1}}{\stackrel{k_{1}}{\rightleftharpoons}} \mathrm{e}^{-}+\mathrm{h}^{+}
$$

(ii) The next step involves the transfer of generated primary hot electrons to the lowest unoccupied molecular orbital (LUMO) of adsorbed bromonucleobases (BrNBs) resulting in the formation of TNI $\left(\mathrm{BrNB}^{*-}\right)$ described by eq 2 .

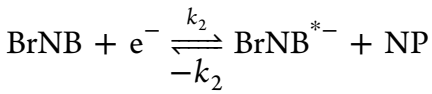

Alternatively, a direct electron transfer (chemical interface damping, CID) could also be possible via a coherent tunnelling process before it interacts with the electron cloud resulting in the formation of the TNI. ${ }^{14,15}$

(iii) Further, the metastable $\mathrm{BrNB}^{*-}$ so formed undergoes cleavage of the $\mathrm{C}-\mathrm{Br}$ bond generating a bromide anion and a nucleobase radical.

$$
\mathrm{BrNB}^{*-} \stackrel{k_{3}}{\rightarrow} \mathrm{NB}^{\bullet}+\mathrm{Br}^{-}
$$

The overall dehalogenation reaction was understood considering a pseudo-first-order reaction rate law. ${ }^{8}$ However, the factors that influence the kinetic rate law, the reaction rate and possible product generation still need to be understood to develop a complete idea of the reaction mechanism. Another important aspect that would affect the reaction rate of plasmon-induced reactions in a heterogeneous medium would be the spatial inhomogeneous distribution of electrons on the surface and hotspots of nanostructures. ${ }^{16}$ It is important to account for these when deriving the kinetic rate equation for all heterogeneous surface reactions induced by plasmons. ${ }^{8,9}$

The probability and mechanism of such surface plasmoninduced reactions depends on several factors namelyfeasibility of hot electron transfer and availability of low lying molecular orbitals of the adsorbate toward TNI formation, ${ }^{3,8,9,17}$ nature and structure of plasmonic substrate, ${ }^{18-22}$ incident photon intensity and excitation energy, $8,20,23-27$ nature of the adsorbates, ${ }^{14,28}$ temperature of metallic nanostructures, ${ }^{25,29,30}$ and so on. Importantly, the availability of hot electrons and their transfer highly depends upon the recovery of metallic atoms from their hot hole counterpart generated by plasmon excitation which determines the success of a plasmon-induced reaction to a great extent. ${ }^{23,31}$ The knowledge of the same would allow one to develop an understanding of different reaction rates observed on different plasmonic substrates based on the adsorbate of interest. ${ }^{8}$

Bromoderivatives of purine nucleobases are potential radiosensitizers in cancer therapy and exhibit a pronounced susceptibility to free and plasmonically generated low energy electrons. ${ }^{32,33}$ We chose them as a model system to understand 
Scheme 1. Schematic Showing the Plasmon-Excitation-Induced Dehalogenation Pathway of Brominated Purines on an Ag Nanostructure and the Varying Observed Rate Constants of the Reaction on the Aggregated Nanostructure (across the HotSpots and the Residual Surface)

Adsorbate perspective

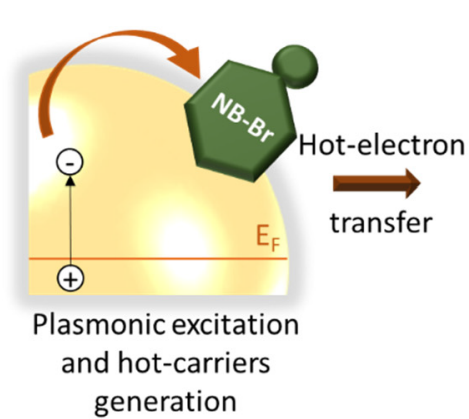
generation

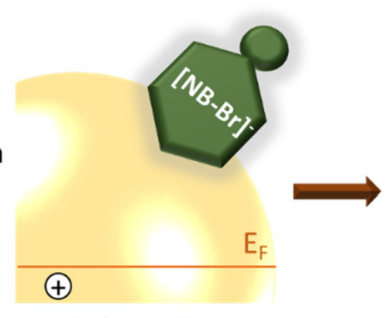

TNI formation the reaction rate law and mechanism of plasmon-induced dehalogenation. The excitation of surface plasmons in aggregates of $\mathrm{Ag}$ and $\mathrm{Au}$ drives the $\mathrm{C}-\mathrm{Br}$ bond cleavage of brominated purines on the metal surface. The reaction rate law will be discussed on the basis of the DEA model described in our previous work $^{8}$ along with addressing the following questions: (i) What parameters determine the reaction rate law? (ii) How is the reaction rate influenced by hot hole deactivation? (iii) What could be the possible dehalogenation products and the plausible role of reaction products and byproducts in the hole-scavenging mechanism? (iv) How does the hot-hole-scavenging mechanism affect the reactivity of purine derivatives on different plasmonic substrates? Next, the plausible mechanism of plasmon-induced dehalogenation reaction will be discussed by elucidating the reaction rate dependence on illumination energy and irradiance. Finally, on the basis of the kinetic data, the reactivity of the two different purine derivatives on $\mathrm{Ag}$ and $\mathrm{Au}$ substrate will be compared. The overall reaction kinetics is monitored in real time using SERS, which allows one to trigger and simultaneously track the reaction with a confocal Raman microscope.

\section{RESULTS AND DISCUSSION}

Plasmon-Induced Dehalogenation Reaction in 8BrGua and 8-BrAde by SERS. The dehalogenation reaction of BrGua adsorbed on AgNPs and AuNPs was carried out under continuous-wave (CW) illumination in ambient condition using a selected laser excitation source. Details of the synthesis of AgNPs and the preparation of the SERS substrate can be found in the Supporting Information (SI). UV-vis absorption spectra of citrate stabilized AgNPs and AuNPs dispersion is shown in Figure S1 (Supporting Information, SI). Field emission scanning electron microscopy (FESEM) of BrGua-coated AgNPs (Figure 1A and Figure S2A,B; SI) and AuNPs (Figure S2C-E; SI) surfaces reveals the aggregated nature of nanostructures. SERS measurements were carried out approximately on the regions shown in the FESEM images (Figure 1A, orange circles, and Figure S2C; SI) to ensure reproducible SERS.

Figure 1B shows the SERS spectra recorded at different time intervals during dissociation of BrGua on AgNPs under CW illumination with a $532 \mathrm{~nm}$ laser. The SERS spectra reveal characteristic sharp peaks at $693 \mathrm{~cm}^{-1}$ (due to a ring breathing mode, solid blue line) along with other peaks at 838 and 1280 $\mathrm{cm}^{-1}$ extracted at $t=0.2 \mathrm{~s}$ (dotted blue line). The cleavage of the $\mathrm{C}-\mathrm{Br}$ bond in BrNBs was ascertained by a decrease in the SERS peak intensity at $693 \mathrm{~cm}^{-1}$ in due course of the reaction time with the concomitant rise of a peak at $670 \mathrm{~cm}^{-1}$, characteristic of the ring breathing mode of Gua (Figure 1B). The peak rising at $1196 \mathrm{~cm}^{-1}$ (dotted lines in pink; Figure 1C) is assigned to a $\mathrm{C} 8-\mathrm{H}$ bending mode $(\delta)$, affirming the Gua formation (via Gua radical intermediate) ${ }^{34}$ supported by additional peaks rising at 834,1007 , and $1627 \mathrm{~cm}^{-1}$. In all cases, the reaction was monitored until saturation of the peak intensity was observed.

Additionally, the SERS spectrum recorded for Gua under the same illumination condition as of BrGua (Figure S3) showed no change in ring breathing mode intensity at $670 \mathrm{~cm}^{-1}$, indicating the stable nature of Gua. All peak assignments have been done with respect to reference SERS frequencies tabulated in ref 34. In general, slight deviations in the wavenumber position of the vibrational modes of BrGua and Gua from the reference SERS values and fluctuations in peak intensity were occasionally observed. This could be attributed to inhomogeneous interparticle gaps resulting in varying structural orientation of the molecules in the aggregates or to local lattice heating. ${ }^{35,36}$ Further details of the experimental results on the dehalogenation reaction of BrAde studied on $\mathrm{Ag}$ aggregates can be found in Figure S4, SI.

Proposed Dehalogenation Reaction Pathway and Kinetic Analysis of BrNBs (BrGua and BrAde) on Ag and Reaction Rate Law. The dehalogenation pathway has been proposed taking into account eqs $1-3$ based on the DEA model $^{8}$ and has been summarized in Scheme 1. Equation 1 involves the generation of hot electrons marked by $<100 \mathrm{fs}$ lifetime. ${ }^{3}$ The plasmon decay $\left(\mathrm{e}^{-}-\mathrm{e}^{-}\right.$scattering) takes place on the fs time scale, and the fraction of effective hot electrons that are generated and transferred remains low with numbers on the order of $10^{-4}-10^{-8} \cdot{ }^{29,37}$ This allows us to consider the formation of TNI in eq 2 to be slow and possibly the rate limiting step. Next, the metastable $\mathrm{BrNB}^{*-}$ so formed undergoes cleavage of the $\mathrm{C}-\mathrm{Br}$ bond generating a bromide anion and a nucleobase radical (eq 2). The dissociation proceeds on a repulsive potential presumably within a single vibrational period (i.e., within tens of $\mathrm{fs}$ ). Hence, this is considered to be the fast step of the dehalogenation reaction. ${ }^{9,11}$

Considering the three elementary steps, the general rate of the dehalogenation of brominated purines can be written as below: 


$$
\text { rate }=\frac{\mathrm{d}[\mathrm{BrNB}]}{\mathrm{d} t}=k_{\mathrm{obs}}[\mathrm{BrNB}]\left[\mathrm{e}^{-}\right]
$$

where $k_{\mathrm{obs}}=\frac{-k_{2} k_{3}}{k_{-2}+k_{3}}$ is the observed rate constant of the reaction. We refer to the SI for a detailed derivation and discussion. Equation 4 reflects that the rate of dehalogenation depends on the concentration of adsorbed BrNBs and effective hot charge carriers directing the dehalogenation kinetics to follow a second order rate law. This could be possible as the illumination area encompasses both hot-spots and remaining surface of the nanostructure, which creates a hot electron concentration gradient in due course of the reaction ${ }^{8,9}$

However, considering a rapid excitation-relaxation process during hot-electron generation under $\mathrm{CW}$ illumination, ${ }^{3}$ the time averaged local concentration of the hot-electrons $\mathrm{e}^{-}$can be considered constant in the initial time. Alternatively, considering the dissociation of the TNI $\left(\mathrm{BrNB}^{*-}\right)$ to be the fast step (as described above), we can write $k_{3} \gg k_{-2}$. These assumptions lead to a simplified pseudo first order eq 5 which can be expressed as below:

$$
\text { rate }=\frac{\mathrm{d}[\mathrm{BrNB}]}{\mathrm{d} t}=k_{\mathrm{obs}}^{\prime}[\mathrm{BrNB}]
$$

where $k^{\prime}{ }_{\text {obs }}=-k_{1} k_{2}[\mathrm{NP}]$ and refers to a pseudo-first-order reaction constant. Detailed considerations can be found in the SI, equations $\mathrm{S} 1-12$.

Therefore, we fit the experimental SERS kinetic data (Figure 1D) with the first-order integrated rate equation (equation S15, SI) obtained from the rate law described in eq 5 . The reaction kinetics was tracked by monitoring the relative decrease in SERS intensity at $693 \mathrm{~cm}^{-1}\left(I_{\text {BrGua }}\right)$ and corresponding rise in peak at $670 \mathrm{~cm}^{-1}\left(I_{\mathrm{Gua}}\right)$ due to product over time $t$ as shown in Figure 1D. Interestingly, a time dependence of $k_{\text {obs }}^{\prime}$ was found (Figure S5, SI) unlike that observed in classical first-order kinetics. This is indicative of the heterogeneous surface reaction that occurs in confined spaces with a limited mobility of the reactants-characteristic of fractal-like reaction kinetics. ${ }^{38}$ It should be mentioned that other factors, such as local temperature and inhomogeneous reaction sites, might affect the reaction rate constant of a heterogeneous reaction taking place in a confined space as well as plasmonic field enhancement in the case of plasmonmediated reactions, as might be the case herein. ${ }^{8,9,38}$

We report on the aggregated nature of the nanostructures (Figure 1A) which results in the generation of inhomogeneous reaction sites. Consequently, the hot spots created in the junction between the NP aggregates serves as confined reaction sites, which exhibit the highest electric field enhancement than on the residual surface. ${ }^{39}$ The presence of such hot spots and the electric field gradient generated across the hot-spot and the residual surface is also confirmed by FDTD simulations, where the geometry was chosen to reproduce an exemplary region (shown in Figure S6A) of BrGua-coated Ag aggregates. The electric field enhancement was simulated for the four different excitation wavelengths, including 488, 532, 633, and $785 \mathrm{~nm}$, as shown in Figure S6BE. The inhomogeneity of the system due to the electric field gradient and hence the time dependence of the reaction rate constant can be explained as follows: in general, a higher reaction rate is expected at the local hot spots than on the rest of the surface due to a higher local concentration of hot electrons. Therefore, within the reaction zone, the gradual change in the local environment in terms of temperature and field enhancement would generate a reaction trajectory, leading to a gradient of reaction constants represented in Scheme 1 on the right-hand side. ${ }^{8}$ The region with higher reaction rate (hotspot) dominates the reaction trajectory in the initial time which is gradually replaced by reaction on the remaining surface that would exhibit lower reaction constant. Therefore, inhomogeneous distribution of the reaction site (substrate perspective) within the illumination zone dominates over the kinetic process (adsorbate perspective), inducing time dependence of the electron transfer step designated by rate constant $k_{2}$.

Therefore, we use the term time-dependent reactioncoefficient $\left(k_{\text {obs }}^{\prime}\right)$ typically used in fractal-like reaction kinetics, ${ }^{38}$ instead of a reaction constant in, for example, (pseudo-) first-order kinetics, which is typically used for a wellstirred mixture of reactants. The time-dependent $k_{\text {obs }}^{\prime}$ is therefore replaced by relation (6) in the electron transfer step (eq 2 ) designated by the rate coefficient $k_{2}$ taking into account the inhomogeneity of the system. ${ }^{38,40}$

$$
k_{2}=k_{\mathrm{f}} t^{-h}, 0 \leq h \leq 1 \text { and } t \geq 1
$$

where $h$ refers to fractal dimension, $t$ refers to time, and $k_{\mathrm{f}}$ refers to the fractal reaction constant which is a timeindependent parameter. Introducing the fractal relation (6) into the pseudo-first-order rate law in eq 5 allows us to obtain the following relation:

$$
[\mathrm{BrGua}]=[\mathrm{BrGua}]_{0} e^{-\left(k_{\mathrm{fl}} \frac{t^{1-h}}{1-h}\right)}
$$

where eq 7 represents the general expression for the pseudofirst-order fractal kinetic equation and $k_{\mathrm{f} 1}$ refers to the reaction constant of the reaction. Detailed derivation of eq 7 is provided in the SI. Herein, we consider [BrGua] and [Gua] to be proportional to the decay and rise in SERS intensity at 693 and $670 \mathrm{~cm}^{-1}$, respectively. Importantly, to exclude the contribution from background noise arising in heterogeneous systems and to achieve improved goodness of the curve fit we consider taking the ratio of SERS signal intensity of $I_{\text {Gua }}$ and $I_{\text {BrGua }}$ obtained in Figure 1D to extract reaction constant $k_{\mathrm{fl}}$, which led to eq 8 . It is to be mentioned that $k_{\mathrm{f} 1}$ will be used as key parameter to discuss all kinetics results and comparison.

$$
\frac{I_{\text {Gua }}}{I_{\text {BrGua }}}=e^{k_{\mathrm{fl}} 1^{1-h} / 1-h}-1
$$

The fitted kinetic data in Figure 2 corresponds to the SERS time trace from BrGua on AgNPs. The pseudo-first-order fractal constant $k_{\mathrm{f} 1}$ extracted from the fit with eq 8 is $0.03 \pm$ $\left(1.3 \times 10^{-4}\right) \mathrm{s}^{-1}$ with $R^{2}=0.952$. A similar trend was observed for time trace data obtained from the dehalogenation reaction observed on BrAde on AgNPs with $633 \mathrm{~nm}$ laser (data not shown).

Next, to comment on the possible dehalogenation products and the role of reaction products and byproducts in the hole deactivation mechanism, we discuss eqs 9 and 10 in further continuation to eqs $1-3$, which has been detailed in the SI and depicted in Scheme 2.

$$
\mathrm{NB}^{\bullet} \stackrel{k_{4}}{\rightarrow} \text { products }
$$

$$
\mathrm{NP}^{*}\left(\mathrm{~h}^{+}\right)+\mathrm{e}^{-} \stackrel{k_{5}}{\rightarrow} \mathrm{NP}
$$




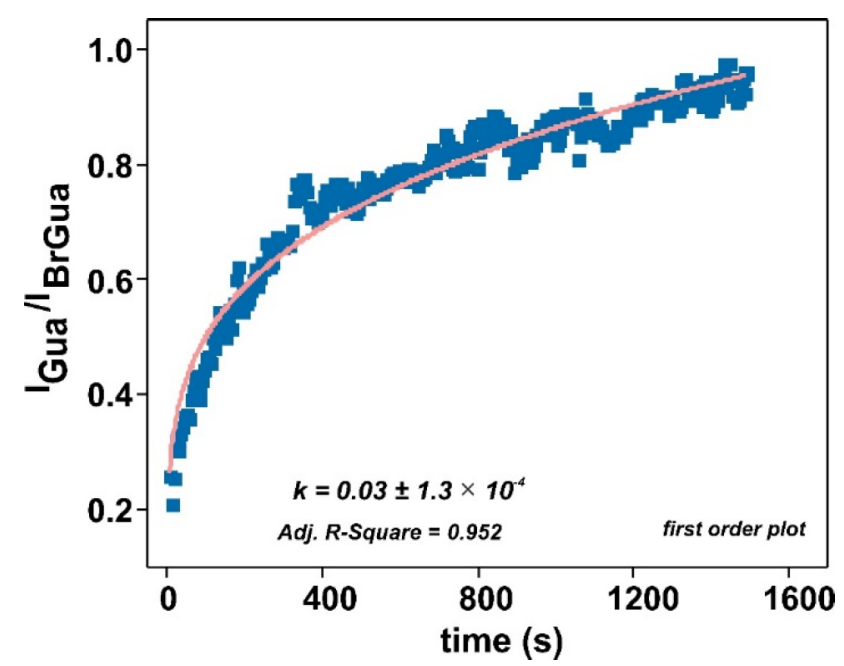

Figure 2. $I_{\mathrm{Gua}} / I_{\mathrm{BrGua}}$ versus time plot when fitted to pseudo-first-order (eq 8 ) integrated fractal rate expression with the pink curve showing the corresponding fit (laser: $633 \mathrm{~nm} ; 1 \mathrm{~mW}, 5 \mathrm{~s}$ ).

The nucleobase radical generated after dehalogenation could translate to form a neutral nucleobase (Gua or Ade) product consuming a $\mathrm{H}^{+}$ion and an additional electron. ${ }^{8}$ Additionally, owing to its high tendency toward oxidation compared to its stable hydrogenated counterpart, it may preferentially follow a one-electron oxidation (eq 11) to generate the oxo-product of guanine and adenine, or it may undergo dimerization to form the dimerized adduct. $^{41,42}$ (refer to equations S20-26, SI)

$$
\mathrm{NB}^{\bullet}+\mathrm{H}_{2} \mathrm{O} \rightarrow \text { 8-oxoNB }+\mathrm{e}^{-}+\mathrm{H}^{+}
$$

For example, the peak arising at around $1460 \mathrm{~cm}^{-1}$ at $6 \mathrm{~s}$ (Figure 1B) can be assigned to vibrational bands characteristic of oxidation of Gua. ${ }^{42} \mathrm{~A}$ very similar feature characteristic of guanine oxidation at around $1610-1620 \mathrm{~cm}^{-1}$ could be seen which can be assigned to the oxo product of Gua, namely, 8oxoguanine or 8 -hydroxoguanine. ${ }^{42,43}$ It should be mentioned that although the vibrational band corresponding to oxidized reaction products can be assigned, a direct quantitative analysis of the oxidized product in the spectral range 1600-1630 and $1400 \mathrm{~cm}^{-1}$ could not be carried out because of their close overlap with the band features of their nonoxidized counterpart. Further, observations made in SERS spectra recorded for the dehalogenation reaction of BrAde (Figure S4A) are included in SI. Importantly, time series normal Raman measurements of BrGua and BrAde were carried out under CW illumination using $532 \mathrm{~nm}$ laser source as a control study (Figure S7A,C). No decay in the intensity of ring breathing mode (or cleavage of the $\mathrm{C}-\mathrm{Br}$ bond) of BrGua and BrAde reflected from Figure $\mathrm{S} 7 \mathrm{~B}$ and $\mathrm{D}$, respectively, was noted, indicating that the bromonucleobases do not undergo decomposition in the absence of plasmonic nanoparticles. That is to say, brominated purines do not show any photoreactivity in the visible range, which is also clear from the absorption spectra recorded for BrGua and BrAde solution (Figure S8A and B), respectively. Additionally, in a recent study, we irradiated 5-bromouracil in the absence of nanoparticles, with focused ns-laser pulses $\left(532 \mathrm{~nm}, 4 \times 10^{9} \mathrm{~W} /\right.$ $\mathrm{cm}^{2}$ ) and observed neither a cleavage of the $\mathrm{C}-\mathrm{Br}$ bond nor any other decomposition of the bromonucleobase. ${ }^{44}$

Like we discussed before, the hole deactivation is an important step, therefore we propose two plausible pathways that can lead to hole deactivation during the dehalogenation reaction (as shown below and refer to equations S22-33, SI). A first plausible hole reduction pathway proposed is the oxidation of the product nucleobase radical (eq 11). The oxidation of the free nucleobases is very well studied in solid electrodes by electrochemical techniques. ${ }^{45,46}$ On a silver electrode, the values for the oxidation of deoxyguanosine $5^{\prime}$ triphosphate (dGTP) start around $0.49 \mathrm{~V}$ vs SHE (Standard hydrogen electrode). ${ }^{47}$ Typically, the triphosphate backbone shifts the oxidation potential of nucleotides toward a more positive potential by almost $0.250 \mathrm{~V}$ compared with the bases. ${ }^{46}$ Therefore, it is likely that the oxidation of Gua takes place around $0.3 \mathrm{~V}$ vs SHE, which indicates toward the consumption of an unstable Gua radical product in the hole reduction, which can undergo oxidation even at a lower

Scheme 2. Schematic Representation of the Overall Plasmon-Induced Dehalogenation Process and Plausible Pathway of Hot Hole Reaction and Fate of Nucleobase Radical (Taking the Example of BrGua) on Ag Surface ${ }^{a}$

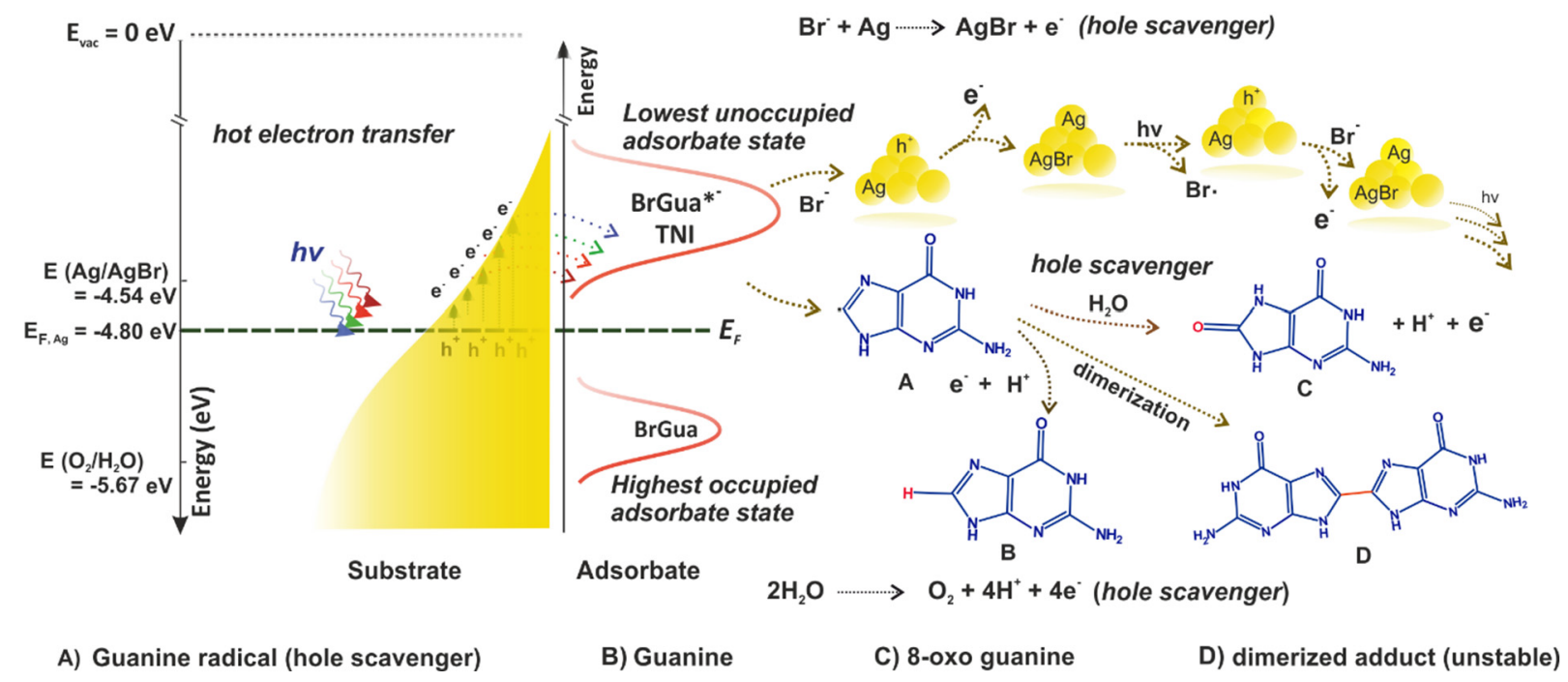

${ }^{a}$ Energy levels shows absolute electrochemical energies, $E(\mathrm{eV})$ with respect to the vacuum level. 

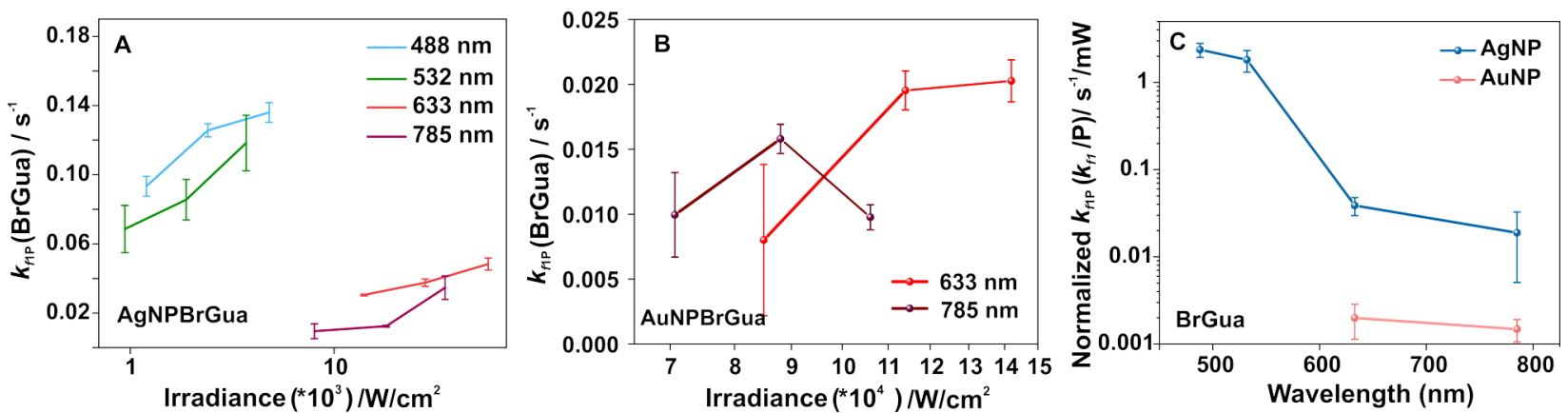

Figure 3. (A) Semilog plot of reaction rate constant plotted as a function of irradiance at different laser wavelength ( $X$-axis is in log scale) determined for dehalogenation reaction of BrGua on (A) AgNPs and (B) AuNPs. Error bar represents the standard deviation of $k_{\mathrm{f} 1}$ values from three different independent measurements carried on different reaction spots. (C) Semilog plot of rate constant $k_{\mathrm{fl}}$ normalized with respect to lowest incident laser power $(P, \mathrm{~mW})$ expressed by $k_{\mathrm{f} 1 P}=k_{\mathrm{f} 1} / P$ plotted at different laser wavelength for dehalogenation of BrGua on AgNPs (blue) and AuNPs (pink), respectively. ( $Y$-axis is in log scale).

potential than $0.3 \mathrm{~V}$ vs SHE. This appears just above the Fermi energy of the AgNP centered around $0.36 \mathrm{~V}$ vs SHE (or, $E$ $(\mathrm{eV})=-4.80 \mathrm{eV}$, absolute electrochemical energy with respect to vacuum given by the relation: $E(\mathrm{eV})=-\left(4.44+E_{\mathrm{SHE}}\right) \mathrm{eV}^{13}$ such that the electrons generated by the oxidation of the base or base radical would be consumed by the hot-holes without any energetic loss. The same can be expected in the case of Ade dehalogenation, considering very small shifts in terms of potential values, since the oxidation of the Ade base also occurs around $0.3 \mathrm{~V}$ vs SHE. ${ }^{47}$ A schematic description of the plasmon-induced dehalogenation process and plausible pathways of hot hole reaction and fate of nucleobase radical product are summarized in Scheme 2.

Second, we propose that the $\mathrm{Br}^{-}$byproduct from the reaction can act as hole scavenger. This involves a favorable one-electron transfer reaction to the hole that occurs at a potential of $0.10 \mathrm{~V}$ vs $\operatorname{SHE}(-4.54 \mathrm{eV})^{48}$ with respect to the Fermi energy of $0.36 \mathrm{~V}$ vs $\operatorname{SHE}(-4.80 \mathrm{eV}) .^{13}$ This would result in the formation of photosensitive $\mathrm{AgBr}$ on the $\mathrm{Ag}$ surface (eq 12) which has also been reported by Xie et al. in one of their seminal works. ${ }^{31}$

$$
\mathrm{Ag}+\mathrm{Br}^{-} \rightarrow \mathrm{AgBr}+\mathrm{e}^{-}
$$

However, because of high photosensitivity (AgI > AgBr > $\mathrm{AgCl}),{ }^{31}$ photodissociation of $\mathrm{AgBr}$ could easily regenerate an $\mathrm{Ag}$ atom and a bromide radical (equation S29, SI) that further engages in stepwise redox processes (refer to SI, equation S30-32 and Scheme 2) to continue the reaction cycle. A third possible pathway for the hole reduction reaction involves water oxidation (equation S23). However, the hole consumption via oxidation of the nucleobase radical and $\mathrm{Br}^{-}$anion proposed to occur on the interface of Ag NPs are more likely to supersede the four-hole oxidation of $\mathrm{H}_{2} \mathrm{O}^{23}(1.23 \mathrm{~V}$ vs SHE $(-5.67 \mathrm{eV})$ ) due to their favorable oxidation potential with respect to Fermi energy.

As assumed in a first approximation in the derivation of the rate equation, the electron transfer to the adsorbate is the ratedetermining step. Nevertheless, in the case of insufficient and slow hole reaction, $k_{5}$ would likely overrule the kinetics of the initial steps marking itself to be the rate-determining step without affecting the kinetic rate laws defined. Having said that, favorable hot hole reaction as discussed above still allows consideration of time-average concentration of hot electrons to be constant under CW illumination. Therefore, we consider a pseudo-first-rate law and rely on the integrated first-order fractal kinetic, eq 8 , to derive further kinetic parameters in the rest of the discussion.

Indeed, that the hole deactivation mechanism is prevalent and places an important role in deciding the reaction rate is further supported by carrying out a dehalogenation reaction of brominated nucleobases on AuNPs. For this, the dehalogenation reaction kinetics were monitored on aggregates of $\mathrm{Ag}$ and $\mathrm{Au}$ at different laser power (irradiance; W/ $\mathrm{cm}^{2}$ ) under different incident photon energy, viz., 488, 532, 633, and 785 $\mathrm{nm}$. Figure $3 \mathrm{~A}$ shows the semilog plot of $k_{\mathrm{f} 1}$ versus irradiance obtained at four different illumination wavelengths (for the reaction monitored in case of BrGua). Reaction rate constant compared at different irradiance (Figure 3A and Figure S9 for BrGua and BrAde on $\mathrm{Ag}$, respectively) showed an increase in value with irradiance at all the wavelengths.

In contrast to the case of $\mathrm{Ag}$, the dehalogenation reaction on $\mathrm{Au}$ could only be followed with 633 and $785 \mathrm{~nm}$ laser illumination. SERS spectra recorded for brominated purines on $\mathrm{Au}$ surfaces under laser illumination at 488 and $532 \mathrm{~nm}$ were dominated by a visible emission background (Figure S10A,B and Figure S11A) which made the traceability of the reaction difficult. This can be explained by radiative recombination attributed to interband transitions in Au that occur in the range of $2.6-2.8 \mathrm{eV}$ close to 488 and $532 \mathrm{~nm}$ laser excitation resulting in plasmon damping (obscuring SERS signal) in addition to off resonance excitation. ${ }^{49}$ The weak Raman scattering signal (ring stretching vibration) from BrAde adsorbed on $\mathrm{Au}$ aggregates when illuminated with the 532 $\mathrm{nm}$ laser is shown in Figure S11A. Further description of kinetics and SERS spectra of brominated purines adsorbed on Au aggregates can be found in SI.

The SERS time traces were fit with eq 8 to obtain the pseudo-first-order kinetic constant for the case of both BrGua and BrAde on the Au substrate (Figure 3B and Figure S12, respectively) under 633 and $785 \mathrm{~nm}$ laser illumination. However, the reaction kinetics observed at laser powers above $6 \mathrm{~mW}$ did not show significant differences in the reaction rate constant (for both BrGua and BrAde when studied with 633 and $785 \mathrm{~nm}$ laser excitation). This is supported by the $k_{\mathrm{f} 1}$ vs irradiance in Figure 3B and Figure S12 unlike that observed in Figure $3 \mathrm{~A}$ and Figure S9. This could possibly be attributed to the small range of laser power selection, which did not allow seeing discernible gradients in the respective reaction coefficients. Nevertheless, the reaction rate observed at $633 \mathrm{~nm}$ was higher than at $785 \mathrm{~nm}$ for both 

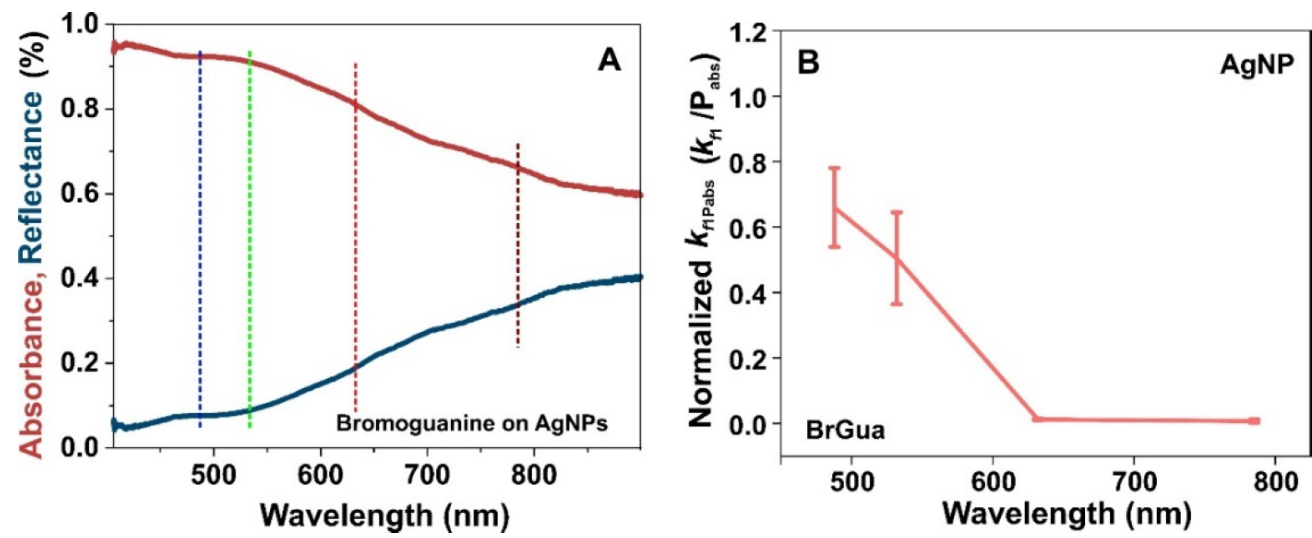

Figure 4. (A) Absorbance and corresponding reflectance spectra recorded on the region shown in Figure S15A for BrGua coated AgNPs. (B) Rate constant $\left(k_{\mathrm{fl}}\right)$ normalized with respect to absorbed laser power $\left(P_{\mathrm{abs}}\right.$ in $\left.\mathrm{mW}\right)$ expressed by $k_{\mathrm{f} 1 P_{\mathrm{abs}}}\left(k_{\mathrm{fl} P_{\mathrm{abs}}}=k_{\mathrm{fl}} / P_{\mathrm{abs}}\right)$ plotted at different laser wavelength for BrGua coated AgNPs. $P_{\text {abs }}=$ incident power $\times$ absorbance. The absorbance of the substrate used for the normalization has been determined from microabsorbance spectra recorded in panel $\mathrm{A}$. The error bar represents the standard deviation of $k_{\mathrm{f} 1}$ values from three different independent measurements.

BrGua and BrAde on $\mathrm{Au}$ albeit less pronounced than for the Ag substrate (summarized in Figure $3 \mathrm{C}$ and Figure S13). The rate constant $\left(k_{\mathrm{f} 1}\right)$ extracted for making the above comparison was normalized with respect to lowest incident laser power $(P$, $\mathrm{mW}$ ) expressed by $k_{\mathrm{f} 1 P}=k_{\mathrm{f} 1} / P$ plotted at different laser wavelengths.

This can be explained by the hole deactivation pathway prevalent on AuNPs surface. In case of $\mathrm{Au}$ also, we consider the one-hole oxidation of $\mathrm{Br}^{-}$(via $\mathrm{AuBr}_{2}{ }^{-}$, eq 13) and oxidation of nucleobases (and their corresponding nucleobase radicals; eq 11), which could serve as a potent hole scavenger along with water oxidation.

$$
\mathrm{Br}^{-} \rightarrow \frac{1}{2} \mathrm{Br}_{2}+\mathrm{e}^{-}
$$

However, unlike $\mathrm{Ag}$, the oxidation potential of $\mathrm{Br}^{-} / \mathrm{Br}_{2}$ (centered around $1.24 \mathrm{~V}$ vs SHE) ${ }^{50}$ and that of nucleobase or nucleobase radical oxidation (centered around $1.20 \mathrm{~V}$ vs $\mathrm{SHE})^{51,52}$ is quite large. This lies close to water oxidation at $1.23 \mathrm{~V}$ vs SHE which might limit the efficiency of hot hole reduction on an $\mathrm{Au}$ surface, thereby diminishing the dehalogenation reaction rate on the $\mathrm{Au}$ surface contrary to what was observed in the case of AgNPs. That is, an inefficient hole deactivation will induce a time delay in the relaxation of the NP system before any subsequent new photoexcitation $\left(\mathrm{e}^{-}-\mathrm{h}^{+}\right.$formation $)$can take place limiting the kinetic rate of dehalogenation on $\mathrm{Au}$ as observed. ${ }^{23}$ In such a situation, any increase in laser power (photon density) will still result in generation of hot carriers which would be available for hotelectron transfer. However, further increase in photon density under such slow hole deactivation rate will lead to a regime which would allow the relaxation of the photo excited carrier via nonradiative $\mathrm{e}^{-}-\mathrm{h}^{+}$recombination (that occurs in ps time scale $)^{53}$ to compete over the hot-electron transfer process. This will limit the number of effective hot-charge carrier available to participate in the dehalogenation reaction resulting in either saturated or diminished value of reaction rate coefficients as reflected in Figure $3 \mathrm{~B}$ or Figure S12. This explains the overall lower reaction rate observed experimentally on the Au surface at 633 and $785 \mathrm{~nm}$ when compared to Ag. A schematic describing the plasmon-induced dehalogenation process and plausible pathways of hot hole reaction and fate of nucleobase radical product on Au surface is presented in Scheme S1. Hence, this draws our attention to the fact that the overall plasmon mediated reaction rate might be limited by the feasibility of hole deactivation. ${ }^{23}$

Importantly, the success of a plasmon-induced reaction (initiated by hot electron transfer) is often questioned either by the existence of a thermally driven reaction pathway or rate acceleration by simple plasmon heating. For an electron-driven photochemical reaction, a linear increase in reaction rate with the laser intensity is expected-that is, rate $\propto$ intensity ${ }^{n}$ with $n$ $=1$, which however deviates to show a super linear dependence with $n>1$ for very high laser intensity or under fs-pulsed laser illumination. ${ }^{26}$ However, a photothermal process shows deviation from such linearity and exhibits an Arrhenius type dependence between the reaction rate and laser intensity for a linear dependence of temperature on the laser power. $^{37}$ The reaction rate coefficient compared at different irradiance (Figure 3A and Figure S9 for BrGua and BrAde on $\mathrm{Ag}$, respectively) shows almost a linear trend although a deviation from linearity is observed at higher irradiance. The steep rise in rate constant observed at the highest irradiance (for some wavelength, viz., 532 and $785 \mathrm{~nm}$ ) could still be the signature of electron-driven photochemical reaction that is mainly observed in extended metallic nanostructures as described in detail by Linic and co-worker in one of their seminal work. ${ }^{26}$ The presence of multiple active hot-spot sites which supports higher rate of hot electron formation at higher photon density results in efficient resonance transfer of electrons to the LUMO of the adsorbate which might be one of the reason for steep rise in reaction rate. Furthermore, a steep rise at the highest irradiance (at different wavelength) could also be due to the dominance of thermal process or plasmon heating. As no clear trend is reflected for both the cases studied, we do not solely depend on reaction rate vs irradiance comparison to analyze the nature of the plasmon mechanism (thermal versus nonthermal). This is because a plasmon-induced reaction which is necessarily initiated via hot electrons might still be influenced photothermally when monitored at high laser power due to localized plasmon heating. ${ }^{25,27}$ Additionally, the microabsorbance spectrum shown in Figure 4A, red band (and Figure S14, yellow band) recorded on the region shown in Figure S15A for 

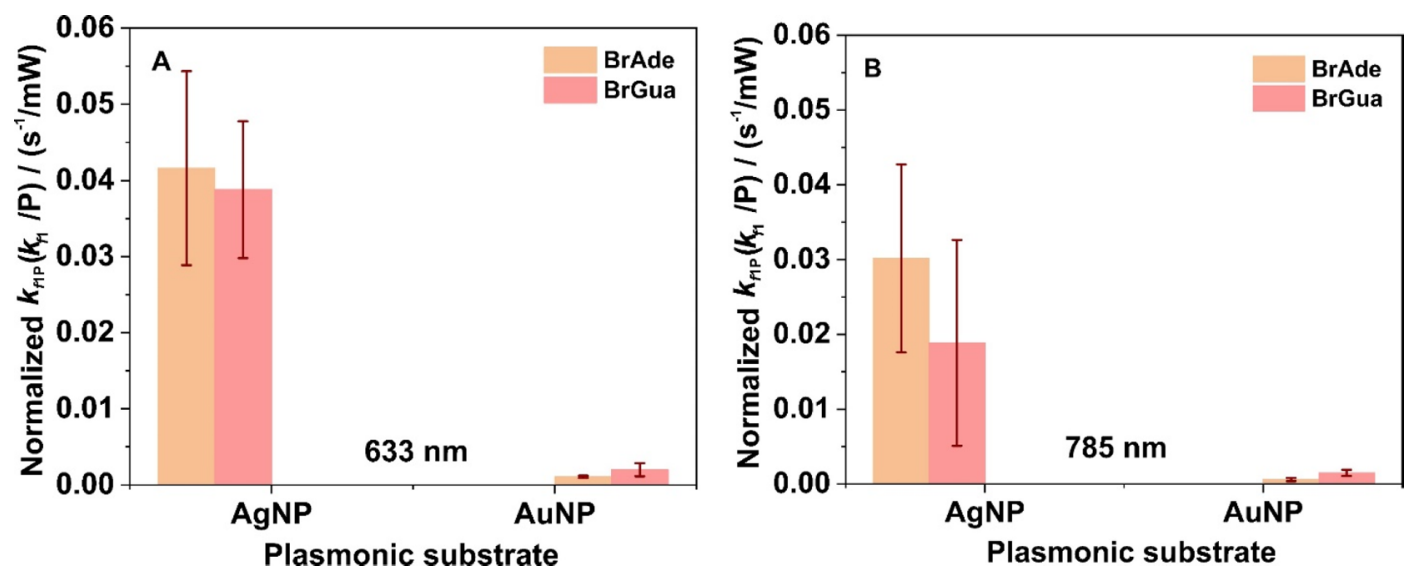

Figure 5. Bar graph showing the comparison of normalized rate constant ( $k_{\mathrm{f} 1 P}$, normalization done with respect to lowest laser power) for dehalogenation of both BrAde and BrGua on AgNPs and AuNPs under (A) $633 \mathrm{~nm}$ and (B) $785 \mathrm{~nm}$ laser excitation. The normalized rate constant of dehalogenation of BrAde is relatively higher than BrGua on AgNPs surface, whereas dehalogenation of BrGua is relatively higher than the rate of dehalogenation of BrAde on AuNPs surface.

BrGua-coated AgNPs (and Figure S15B for BrAde) shows pronounced absorbance at $488 \mathrm{~nm}$. This could also lead to a temperature increase aiding the reaction thermally.

Further, we compare the reaction rate constant normalized to the absorbed power under different laser excitation wavelengths to comment on the role of hot electrons in plasmon-induced dehalogenation. The rate constant $\left(k_{\mathrm{f} 1}\right)$ normalized with respect to the absorbed power $P_{\mathrm{abs}}$ given by the expression $k_{\mathrm{f} 1 P_{\mathrm{abs}}}\left(k_{\mathrm{f} 1 P_{\mathrm{abs}}}=k_{\mathrm{fl}} / P_{\mathrm{abs}}\right.$, where $P_{\mathrm{abs}}=$ Incident Power $\times$ Absorbance $(\lambda)$ ) plotted at different wavelengths showed an increasing trend with increase in laser excitation energy following the order ${ }^{\text {BrGua }} k_{488}>{ }^{\text {BrGua }} k_{532}>{ }^{\text {BrGua }} k_{633}>$ ${ }^{\text {BrGua }} k_{785}$ (Figure 4B). The microabsorbance spectrum in Figure $4 \mathrm{~A}$ showed a decrease in absorbance with an increase in wavelength; and, the field enhancement calculated according to the FDTD simulations (determined on an exemplary regions of BrGua coated $\mathrm{Ag}$ aggregates) follows the order: $E_{488}$ $>E_{532}>E_{633}>E_{785}$ (Figure S6, refer to SI for details). The experimental reflectance spectrum (Figure 4A, blue curve) also closely resembles the simulated reflectance spectrum shown in Figure S16. Typically, plasmon-induced reactions observed on regions that correspond to higher absorbance and high field enhancement should exhibit higher reactivity because of the higher rate of hot electron generation under resonant illumination. $^{20,27}$ Therefore, highly pronounced absorption (indicated by dotted colored lines - blue and green, Figure 4A) and comparatively high field enhancement at 488 and $532 \mathrm{~nm}$ (Figure S6B and C, SI) would result in buildup of population of hot carriers in this regime explaining the observed trend in reactivity. On the other hand, off resonance and lower optical absorption at 633 and $785 \mathrm{~nm}$ under low photon energy on $\mathrm{Ag}$ substrate and lower plasmonic field enhancement (Figure S6D and E, SI) explains the corresponding lower reaction rate observed. A very similar argument holds true in case of dehalogenation kinetics for BrAde on AgNPs (refer to Figure S14).

Next, in the case of nonthermal or hot electron-driven processes, the energy distribution of the hot carriers depends upon the excitation wavelength as high energy photons can excite electrons far from the Fermi level compared with lowerenergy photons. ${ }^{17,27}$ Based on the decreasing energy of hot carriers with increasing wavelength, a significant drop in reaction rate should be observed in the case of a hot electrondriven process. Furthermore, temperature rise of a system depends upon the amount of absorbed optical energy. ${ }^{2}$ Therefore, in case of a nonthermal process, the reaction rate normalized to the absorbed number of photons should show a wavelength dependence with maxima in the spectral range where hot electron generation is efficient. ${ }^{27} \mathrm{~A}$ clear dependence of reaction coefficient normalized to absorbed power versus wavelength can be observed in Figure $4 \mathrm{~B}$ and Figure $\mathrm{S} 17$ with maxima at the SPR region of $\mathrm{Ag}$ nanoparticles in the case of both BrGua and BrAde, respectively. This allowed us to conclude that the dehalogenation reaction is driven by hotelectron indicating toward the nonthermal nature of the reaction. However, we do not completely eliminate the thermal activation of reactants and hot charge carriers with increase in irradiance and laser energy.

Additionally, we considered analyzing the relative SERS response of the brominated purines on the surface of $\mathrm{Ag}$ and Au. Therefore, a histogram plot showing relative normalized SERS intensity of BrAde and BrGua on the aggregated surface of AgNPs and AuNPs under 633 and $785 \mathrm{~nm}$ laser excitation is shown in Figure S18. We arrive at a conclusion that the SERS sensitivity of BrAde is higher compared with BrGua on both $\mathrm{Ag}$ and $\mathrm{Au}$ substrate. Also, $\mathrm{Ag}$ appears to be a better SERS substrate for both BrAde and BrGua than $\mathrm{Au}$, which was apparently clear from Figure 3C and Figure S13.

Lastly, we took interest to know whether the observed kinetics is dependent upon the nature of brominated purines involved. Overall, our findings showed comparatively higher reactivity of BrAde on AgNPs than BrGua, which reverses its reactivity on the AuNP surface under both the laser illumination at 632 and $785 \mathrm{~nm}$ independent of their relative SERS sensitivity response. This is shown by the bar diagrams in Figure $5 \mathrm{~A}, \mathrm{~B}$ of the rate constant $\left(k_{\mathrm{f} 1}\right)$ normalized with respect to incident laser power $(P)$ expressed by $k_{\mathrm{f} 1 P}$, although an explanation for the reactivity of brominated purines on the respective plasmonic substrates demands a separate study as a whole. However, a preliminary idea of the higher reactivity of BrGua over BrAde on the AuNPs surface could be drawn taking into account the binding affinity of the respective unsubstituted nucleobases on $\mathrm{Au}$ and AgNPs and shift in density of states upon surface adsorption. ${ }^{54-58}$ A plausible reasoning for the observation has been discussed in SI. 


\section{CONCLUSIONS}

In summary, hot-electron-induced cleavage of $\mathrm{C}-\mathrm{Br}$ bonds, release of $\mathrm{Br}^{-}$, and formation of a reactive radical intermediate are proposed to be the essential steps that form the basis of the kinetic rate equation. Based on a proposed kinetic model, the time-average constant concentration of hot electrons and time scale of the dissociation of transient negative ions (TNI) is considered to play a critical role in defining the rate law. Therefore, a pseudo-first-order dehalogenation kinetics has been implemented considering the time-averaged concentration of hot electrons to be constant. Importantly, $\mathrm{Br}^{-}$and product nucleobase radical play an important role in hole deactivation which ultimately guides the dehalogenation rate. Although the reaction rate is initially suggested to be limited by electron transfer to the LUMO of adsorbed BrNBs, we arrive at the conclusion that insufficient hole deactivation could impede the reaction rate significantly marking itself as ratedetermining step for the overall reaction. The favorable oxidation potential of $\mathrm{Br}^{-}$and the nucleobases radicals toward hole reaction explains the overall higher reactivity of dehalogenation kinetics observed on $\mathrm{Ag}$, which is compromised on Au surfaces because of the large oxidation potential of both $\mathrm{Br}^{-}$and the nucleobase radical.

Higher reaction rate observed at the higher laser energy has been explained by pronounced surface plasmon resonance (SPR) absorption accompanied by high plasmonic enhancement in the case of Ag compared with $\mathrm{Au}$, which results in the generation of higher concentration of hot carriers. We further conclude on the hot-electron-driven nature of the dehalogenation reaction over the thermal nature by showing the wavelength dependency of the reaction rate normalized to absorbed optical power. The dehalogenation reaction rate law is independent of the nature of brominated nucleobases and the plasmonic substrate, although the feasibility of the reaction and their corresponding reaction rate is guided by nature of the brominated purines and the plasmonic substrate involved. Based on the present findings, future implications lie in further monitoring of hot-electron-induced reactions in brominated purines when incorporated in long ss and ds DNA by the SERS method. ${ }^{59}$ The success of which would direct one to employ the bromo-derivative of the purines and other potent electrophilic radiosensitizers for real time study in cellular environment for upgraded cancer therapy.

\section{MATERIALS AND METHODS}

Chemicals. Guanine (Gua), 8-bromoguanine (BrGua), silver nitrate $\left(\mathrm{AgNO}_{3}\right)$, and trisodium citrate were purchased from Sigma-Aldrich (Germany). 8-Bromoadenine (BrAde) was purchased from Carbosynth (U.K.). Sodium hydroxide $(\mathrm{NaOH})$ was purchased from Merck. AuNPs (diameter 40 $\mathrm{nm}$ ) were purchased from BBI solutions. Si-wafers were purchased from CrysTec $\mathrm{GmbH}$ (100 mm, p-Typ). All chemicals were used as received without further purification. Milli-Q grade water was used for synthesis and experimental work.

Synthesis of AgNPs. AgNPs were synthesized following Lee and Meisel protocol ${ }^{60}$ with some modifications. Briefly, 1 $\mathrm{mM} \mathrm{AgNO}_{3}$ dissolved in $500 \mathrm{~mL}$ of Milli-Q water were heated up to boil under reflux condition at $125{ }^{\circ} \mathrm{C}$ with constant stirring $(600 \mathrm{rpm})$. This was followed by addition of $10 \mathrm{~mL}$ of $155 \mathrm{mM}$ sodium citrate to the same solution using a syringe and the boiling was continued for another $1 \mathrm{~h}$. The formation of AgNPs was ensured by the appearance of final brown-yellow color of the dispersion. Detailed characterization of as synthesized AgNP dispersion has already been presented in ref 34 .

\section{SERS STUDY}

Sample Preparation. Citrate (cit) stabilized Ag and Au NPs were first functionalized with brominated analogue of purine derivative, viz., BrAde and BrGua. ${ }^{34}$

AgNPs. In the first step, $1 \mathrm{~mL}$ of as-synthesized AgNP dispersion was purified by centrifugation at $2000 \mathrm{rcf}$ for $10 \mathrm{~min}$ at $20^{\circ} \mathrm{C}$, and the precipitate was redispersed in $1 \mathrm{~mL}$ of MilliQ water. Next, $20 \mu \mathrm{L}$ of $0.5 \mathrm{mM}$ solution of Gua and their brominated analogue of Gua and Ade were mixed with $80 \mathrm{uL}$ of centrifuged AgNPs dispersion and allowed to incubate for 1 $\mathrm{h}$ at room temperature. Because of poor water solubility, 0.5 $\mathrm{mM}$ of Gua and BrGua were made in $\mathrm{NaOH}$ solution such that the final concentration of $\mathrm{NaOH}$ is $13 \mathrm{mM}$ and $0.8 \mathrm{mM}$ respectively. Upon $1 \mathrm{~h}$ of incubation, the resulting mixture was centrifuged at $1000 \mathrm{rcf}$ for $10 \mathrm{~min}$ at $20{ }^{\circ} \mathrm{C}(\times 2)$, and the precipitate was redispersed in $20 \mu \mathrm{L}$ Milli-Q water. Immediately, $10 \mu \mathrm{L}$ of the sample dispersion was drop cast on a cleaned Si-substrate and was left for overnight drying at ambient condition.

AuNPs. The AuNP samples for SERS measurements were prepared by similar method except for few changes as stated. Then $1 \mathrm{~mL}$ of AuNP dispersion was purified by centrifugation at $2000 \mathrm{rcf}$ for $10 \mathrm{~min}$ at $20{ }^{\circ} \mathrm{C}$, and the precipitate was redispersed in $1 \mathrm{~mL}$ of Milli-Q water. Next, $20 \mu \mathrm{L}$ of $0.5 \mathrm{mM}$ solution of brominated analogue of Ade and Gua were mixed with $80 \mu \mathrm{L}$ of centrifuged AuNPs dispersion and allowed to incubate for $1 \mathrm{~h}$ at room temperature. Due to poor water solubility, $0.5 \mathrm{mM}$ of BrGua were made in $\mathrm{NaOH}$ solution such that the final concentration of $\mathrm{NaOH}$ is 2 and $4.4 \mathrm{mM}$ respectively. Upon $1 \mathrm{~h}$ of incubation, the resulting mixture was centrifuged at $1000 \mathrm{rcf}$ for $10 \mathrm{~min}$ at $10{ }^{\circ} \mathrm{C}(\times 2)$, and the precipitate was redispersed in $20 \mu \mathrm{L}$ Milli-Q water. Immediately, $10 \mu \mathrm{L}$ of the sample dispersion was drop cast on a cleaned Si-substrate and was left for overnight drying in ambient condition.

Upon treatment, the brominated derivatives of purines preferentially get adsorbed on the surface of NPs, replacing the citrate as surface stabilizers meanwhile inducing aggregation in the dispersion. ${ }^{54}$ This is further facilitated during the two-step centrifugation process, which helped remove the citrate layer from $\mathrm{Ag}$ and AuNPs and simultaneously inducing aggregation of NPs via intermolecular $\mathrm{H}$-bonds or by $\pi-\pi^{*}$ stacking interaction between the brominated analogues. ${ }^{34,55,61}$ The extent of induced aggregation depends upon the type of brominated nucleobases and their respective binding energies toward NPs.

SERS Kinetic Measurements. Kinetic measurements of brominated purines treated AgNPs and AuNPs were carried out using WITec alpha300 Raman-microscope equipped with four different excitation laser sources $(488,532,632$, and 785 $\mathrm{nm}$ ) under a $10 \times$ objective $(\mathrm{NA}=0.25)$. The laser power, integration, and accumulation time for each measurement employed can be found as stated where necessary. All measurements were carried out in triplicate in different sample areas. All SERS spectra obtained were processed using WITec Project 5 and Origin 9.1 software.

Microabsorbance Measurements. The measurements were performed with a microabsorbance spectrometer, which 
is described elsewhere in detail. ${ }^{62}$ Briefly, light from a supercontinuum laser (Fianium, SC-400-4) was guided through a beamsplitter toward a modified microscope and focused on the sample with an Olympus SLMPlan FLN 20x objective with an NA of 0.25 . The reflected light was collected by the same objective and guided by an optical fiber (Oceans optics QP600-2-UV-BX) to the spectrometer (Avantes, Avaspec 3648). The microabsorbance spectra were measured at characteristic places that were similar to the SERS measurement spots. The reflectance of the Si substrate close to the measurement position was used as a reference $I_{\text {Reference }}$ for reflection. The reflectance was calculated as

$$
R=\frac{I_{\text {Sample }}-I_{\text {Dark }}}{I_{\text {Reference }}-I_{\text {Dark }}}
$$

The absorbance was determined as $A=1-R$, since transmission was prevented by the $\mathrm{Si}$ substrate.

Finite-Difference Time-Domain Method (FDTD). FDTD simulations of AgNP on a $\mathrm{Si}$ substrate have been carried out using the software Lumerical FDTD Solutions 8.6.3 with a mesh size of $1 \mathrm{~nm}$. The Si substrate was covered with a $2 \mathrm{~nm} \mathrm{SiO}$ layer and decorated with cylindrical $40 \mathrm{~nm}$ AgNPs imported from SEM images of the sample. A linear polarized plane wave source ( 400 to $900 \mathrm{~nm}$ ) irradiated the sample from the top and a power monitor above the light source recorded the reflectance of the sample. The E-field was monitored in the $x y$-plane in the center of the AgNPs.

\section{ASSOCIATED CONTENT}

\section{SI Supporting Information}

The Supporting Information is available free of charge at https://pubs.acs.org/doi/10.1021/acscatal.1c01851.

Detailed derivation of reaction rate law; integrated rate equation; stepwise redox reaction involved in hot-hole reaction; UV-vis spectra of $\mathrm{BrGa}$ and BrAde solution and $\mathrm{Ag}$ and AuNP dispersion; FESEM images of BrGua coated aggregates of AgNPs and AuNPs; normal Raman time-series spectra of BrGua and BrAde; time-series SERS spectra of BrAde on AgNPs and SERS spectra of bromoderivatives of purines on AuNPs; corresponding kinetics rate constant versus time plot; kinetic rate constant plot at different wavelength and power; plot of normalized rate constant with respect to power and absorbed power at different wavelength of BrAde on $\mathrm{Ag}$ and AuNPs; normalized SERS intensity plot of BrGua and BrAde on $\mathrm{Ag}$ and $\mathrm{Au}$; microabsorbance-reflectance plot of BrAde coated AgNPs; FDTD simulation of field enhancement values at different wavelength and 3DFDTD simulated reflectance spectra (PDF)

\section{AUTHOR INFORMATION}

\section{Corresponding Author}

Ilko Bald - Institute of Chemistry, Physical Chemistry, University of Potsdam, 14476 Potsdam, Germany; (1) orcid.org/0000-0002-6683-5065; Email: ilko.bald@unipotsdam.de

\section{Authors}

Anushree Dutta - Institute of Chemistry, Physical Chemistry, University of Potsdam, 14476 Potsdam, Germany; (ㄷ) orcid.org/0000-0001-5862-3233
Robin Schürmann - Institute of Chemistry, Physical Chemistry, University of Potsdam, 14476 Potsdam, Germany

Sergio Kogikoski, Jr. - Institute of Chemistry, Physical Chemistry, University of Potsdam, 14476 Potsdam, Germany

Niclas S. Mueller - Department of Physics, Freie Universität Berlin, 14195 Berlin, Germany; ㅇo orcid.org/0000-00028688-1974

Stephanie Reich - Department of Physics, Freie Universität Berlin, 14195 Berlin, Germany; 이이이.org/0000-00022391-0256

Complete contact information is available at:

https://pubs.acs.org/10.1021/acscatal.1c01851

\section{Notes}

The authors declare no competing financial interest.

\section{ACKNOWLEDGMENTS}

We thank Dr. Frank Jaiser for recording the FESEM images. This research was supported by the European Research Council (ERC; consolidator grant no. 772752). S.R and N.S.M were supported by the European Research Council (ERC) within the project DarkSERS (772108) and the Focus Area NanoScale of Freie Universitaet Berlin.

\section{REFERENCES}

(1) Zhang, Y.; He, S.; Guo, W.; Hu, Y.; Huang, J.; Mulcahy, J. R.; Wei, W. D. Surface-Plasmon-Driven Hot Electron Photochemistry. Chem. Rev. 2018, 118, 2927-2954.

(2) Brooks, J. L.; Warkentin, C. L.; Saha, D.; Keller, E. L.; Frontiera, R. R. Toward a Mechanistic Understanding of Plasmon-Mediated Photocatalysis. Nanophotonics 2018, 7, 1697-1724.

(3) Zhang, Z.; Zhang, C.; Zheng, H.; Xu, H. Plasmon-Driven Catalysis on Molecules and Nanomaterials. Acc. Chem. Res. 2019, 52, $2506-2515$

(4) Manjavacas, A.; Liu, J. G.; Kulkarni, V.; Nordlander, P. PlasmonInduced Hot Carriers in Metallic Nanoparticles. ACS Nano 2014, 8, $7630-7638$.

(5) Chang, L.; Besteiro, L. V.; Sun, J.; Santiago, E. Y.; Gray, S. K.; Wang, Z.; Govorov, A. O. Electronic Structure of the Plasmons in Metal Nanocrystals: Fundamental Limitations for the Energy Efficiency of Hot Electron Generation. ACS Energy Lett. 2019, 4, $2552-2568$

(6) Zhan, C.; Chen, X. J.; Huang, Y. F.; Wu, D. Y.; Tian, Z. Q. Plasmon-Mediated Chemical Reactions on Nanostructures Unveiled by Surface-Enhanced Raman Spectroscopy. Acc. Chem. Res. 2019, 52, 2784-2792.

(7) Feng, H. S.; Dong, F.; Su, H. S.; Sartin, M. M.; Ren, B. In Situ Investigation of Hot-Electron-Induced Suzuki-Miyaura Reaction by Surface-Enhanced Raman Spectroscopy. J. Appl. Phys. 2020, 128, 173105.

(8) Schürmann, R.; Bald, I. Real-Time Monitoring of Plasmon Induced Dissociative Electron Transfer to the Potential DNA Radiosensitizer 8-Bromoadenine. Nanoscale 2017, 9, 1951-1955.

(9) Linic, S.; Christopher, P.; Ingram, D. B. Plasmonic-Metal Nanostructures for Efficient Conversion of Solar to Chemical Energy. Nat. Mater. 2011, 10, 911-921.

(10) Ho, W. Reactions at Metal Surfaces Induced by Femtosecond Lasers, Tunneling Electrons, and Heating. J. Phys. Chem. 1996, 100, 13050-13060.

(11) Christopher, P.; Xin, H.; Linic, S. Visible-Light-Enhanced Catalytic Oxidation Reactions on Plasmonic Silver Nanostructures. Nat. Chem. 2011, 3, 467-472.

(12) Mukherjee, S.; Libisch, F.; Large, N.; Neumann, O.; Brown, L. V.; Cheng, J.; Lassiter, B.; Carter, E. A.; Nordlander, P.; Halas, N. J. Hot Electrons Do the Impossible: Plasmon-Induced Dissociation of $\mathrm{H}_{2}$ on Au. Nano Lett. 2013, 13, 240-247. 
(13) Liu, J.; Cai, Z. Y.; Sun, W. X.; Wang, J. Z.; Shen, X. R.; Zhan, C.; Devasenathipathy, R.; Zhou, J. Z.; Wu, D. Y.; Mao, B. W.; Tian, Z. Q. Plasmonic Hot Electron-Mediated Hydrodehalogenation Kinetics on Nanostructured Ag Electrodes. J. Am. Chem. Soc. 2020, 142, 17489-17498.

(14) Boerigter, C.; Aslam, U.; Linic, S. Mechanism of Charge Transfer from Plasmonic Nanostructures to Chemically Attached Materials. ACS Nano 2016, 10, 6108-6115.

(15) Kazuma, E.; Jung, J.; Ueba, H.; Trenary, M.; Kim, Y. Real-space and real-time observation of a plasmon-induced chemical reaction of a single molecule. Science 2018, 360, 521-526.

(16) Linic, S.; Chavez, S.; Elias, R. Flow and Extraction of Energy and Charge Carriers in Hybrid Plasmonic Nanostructures. Nat. Mater. 2021, DOI: $10.1038 / \mathrm{s} 41563-020-00858-4$.

(17) Yu, Y.; Wijesekara, K. D.; Xi, X.; Willets, K. A. Quantifying Wavelength-Dependent Plasmonic Hot Carrier Energy Distributions at Metal/Semiconductor Interfaces. ACS Nano 2019, 13, 3629-3637.

(18) Mueller, N. S.; Vieira, B. G. M.; Hoing, D.; Schulz, F.; Barros, E. B.; Lange, H.; Reich, S. Direct optical excitation of dark plasmons for hot electron generation. Faraday Discuss. 2019, 214, 159-173.

(19) Mukherjee, S.; Zhou, L.; Goodman, A. M.; Large, N.; AyalaOrozco, C.; Zhang, Y.; Nordlander, P.; Halas, N. J. Hot-ElectronInduced Dissociation of $\mathrm{H}_{2}$ on Gold Nanoparticles Supported on $\mathrm{SiO}_{2}$. J. Am. Chem. Soc. 2014, 136, 64-67.

(20) Schürmann, R.; Ebel, K.; Nicolas, C.; Milosavljević, A. R.; Bald, I. Role of Valence Band States and Plasmonic Enhancement in Electron-Transfer-Induced Transformation of Nitrothiophenol. J. Phys. Chem. Lett. 2019, 10, 3153-3158.

(21) Cortés, E.; Xie, W.; Cambiasso, J.; Jermyn, A. S.; Sundararaman, R.; Narang, P.; Schlücker, S.; Maier, S. A. Plasmonic Hot Electron Transport Drives Nano-Localized Chemistry. Nat. Commun. 2017, 8, 1-10.

(22) Pal, S.; Dutta, A.; Paul, M.; Chattopadhyay, A. PlasmonEnhanced Chemical Reaction at the Hot Spots of End-to-End Assembled Gold Nanorods. J. Phys. Chem. C 2020, 124, 3204-3210.

(23) Kim, Y.; Smith, J. G.; Jain, P. K. Harvesting Multiple ElectronHole Pairs Generated through Plasmonic Excitation of Au Nanoparticles. Nat. Chem. 2018, 10, 763-769.

(24) Zhou, L.; Swearer, D. F.; Zhang, C.; Robatjazi, H.; Zhao, H.; Henderson, L.; Dong, L.; Christopher, P.; Carter, E. A.; Nordlander, P.; Halas, N. J. Quantifying Hot Carrier and Thermal Contributions in Plasmonic Photocatalysis. Science 2018, 362, 69-72.

(25) Koopman, W.; Sarhan, R. M.; Stete, F.; Schmitt, C. N. Z.; Bargheer, M. Decoding the Kinetic Limitations of Plasmon Catalysis: The Case of 4-Nitrothiophenol Dimerization. Nanoscale 2020, 12, 24411-24418.

(26) Christopher, P.; Xin, H.; Marimuthu, A.; Linic, S. Singular Characteristics and Unique Chemical Bond Activation Mechanisms of Photocatalytic Reactions on Plasmonic Nanostructures. Nat. Mater. 2012, 11, 1044-1050.

(27) Rodio, M.; Graf, M.; Schulz, F.; Mueller, N. S.; Eich, M.; Lange, $\mathrm{H}$. Experimental Evidence for Nonthermal Contributions to PlasmonEnhanced Electrochemical Oxidation Reactions. ACS Catal. 2020, 10, 2345-2353.

(28) Foerster, B.; Spata, V. A.; Carter, E. A.; Sönnichsen, C.; Link, S. Plasmon Damping Depends on the Chemical Nature of the Nanoparticle Interface. Sci. Adv. 2019, 5, eaav0704.

(29) Dubi, Y.; Un, I. W.; Sivan, Y. Thermal Effects - an Alternative Mechanism for Plasmon-Assisted Photocatalysis. Chem. Sci. 2020, 11, 5017-5027.

(30) Jain, P. K. Taking the Heat off of Plasmonic Chemistry. J. Phys. Chem. C 2019, 123, 24347-24351.

(31) Xie, W.; Schlücker, S. Hot Electron-Induced Reduction of Small Molecules on Photorecycling Metal Surfaces. Nat. Commun. 2015, 6, 7570.

(32) Schürmann, R.; Tsering, T.; Tanzer, K.; Denifl, S.; Kumar, S. V. K.; Bald, I. Resonant Formation of Strand Breaks in Sensitized Oligonucleotides Induced by Low-Energy Electrons $(0.5-9 \mathrm{eV})$. Angew. Chem., Int. Ed. 2017, 56, 10952.
(33) Schürmann, R.; Vogel, S.; Ebel, K.; Bald, I. The physicochemical basis of DNA radiosensitization - Implications for cancer radiation Therapy. Chem. - Eur. J. 2018, 24, 10271-10279.

(34) Dutta, A.; Schürmann, R.; Bald, I. Plasmon Mediated Decomposition of Brominated Nucleobases on Silver Nanoparticles - A Surface Enhanced Raman Scattering (SERS) Study. Eur. Phys. J. D 2020, 74, 1-9.

(35) King, M. D.; Khadka, S.; Craig, G. A.; Mason, M. D. Effect of Local Heating on the SERS Efficiency of Optically Trapped Prismatic Nanoparticles. J. Phys. Chem. C 2008, 112, 11751-11757.

(36) Micic, M.; Klymyshyn, N.; Lu, H. P. Finite Element Method Simulations of the Near-Field Enhancement at the Vicinity of Fractal Rough Metallic Surfaces. J. Phys. Chem. B 2004, 108, 2939-2947.

(37) Baffou, G.; Bordacchini, I.; Baldi, A.; Quidant, R. Simple Experimental Procedures to Distinguish Photothermal from HotCarrier Processes in Plasmonics. Light: Sci. Appl. 2020, 9, 108.

(38) Kopelman, R. Fractal Reaction Kinetics. Science 1988, 241, $1620-1626$.

(39) Le Ru, E. C.; Etchegoin, P. G.; Meyer, M. Enhancement Factor Distribution around A single Surface-Enhanced Raman Scattering Hot Spot and Its Relation to Single Molecule detection. J. Chem. Phys. 2006, 125, 204701.

(40) Sieland, F.; Schneider, J.; Bahnemann, D. W. Fractal Charge Carrier Kinetics in TiO2. J. Phys. Chem. C 2017, 121, 24282-24291.

(41) Li, Q.; Batchelor-Mcauley, C.; Compton, R. G. Electrochemical Oxidation of Guanine: Electrode Reaction Mechanism and Tailoring Carbon Electrode Surfaces to Switch between Adsorptive and Diffusional Responses. J. Phys. Chem. B 2010, 114, 7423-7428.

(42) Ibañez, D.; Santidrian, A.; Heras, A.; Kalbáć, M.; Colina, A. Study of Adenine and Guanine Oxidation Mechanism by SurfaceEnhanced Raman Spectroelectrochemistry. J. Phys. Chem. C 2015, 119, 8191-8198.

(43) D’Amico, F.; Cammisuli, F.; Addobbati, R.; Rizzardi, C.; Gessini, A.; Masciovecchio, C.; Rossi, B.; Pascolo, L. Oxidative Damage in DNA Bases Revealed by UV Resonant Raman Spectroscopy. Analyst 2015, 140, 1477-1485.

(44) Marques, T. S.; Schürmann, R.; Ebel, K.; Heck, C.; Smiałek, M. A.; Eden, S.; Mason, N.; Bald, I. Kinetics of molecular decomposition under irradiation of gold nanoparticles with nanosecond laser pulses-A 5-Bromouracil case study. J. Chem. Phys. 2020, 152, 124712.

(45) Chiorcea-Paquim, A. M.; Oliveira-Brett, A. M. Nanostructured Material-Based Electrochemical Sensing of Oxidative DNA Damage Biomarkers 8-Oxoguanine and 8-Oxodeoxyguanosine: A Comprehensive Review. Microchim. Acta 2021, 188, 1-13.

(46) Chiorcea-Paquim, A.-M.; Oliveira-Brett, A. M. DNA Electrochemical Biosensors for In Situ Probing of Pharmaceutical Drug Oxidative DNA Damage. Sensors 2021, 21, 1125.

(47) Fan, C.; Song, H.; Hu, X.; Li, G.; Zhu, J.; Xu, X.; Zhu, D. Voltammetric Response and Determination of DNA with a Silver Electrode. Anal. Biochem. 1999, 271, 1-7.

(48) Rezaei-Zarchi, S.; Saboury, A. A.; Norouzi, P.; Hong, J.; Barzegar, A.; Ganjali, M. R.; Ghourchian, H.; Moosavi-Movahedi, A. A.; Javed, A.; Rostami, A. A. Electrochemical Recognition of Metalloproteins by Bromide-Modified Silver Electrode - A New Method. Int. J. Mol. Sci. 2007, 8, 723-735.

(49) Zhang, X.; Huang, C.; Wang, M.; Huang, P.; He, X.; Wei, Z. Transient Localized Surface Plasmon Induced by Femtosecond Interband Excitation in Gold Nanoparticles. Sci. Rep. 2018, 8, 1-7.

(50) Tariq, M. Electrochemistry of $\mathrm{Br}^{-} / \mathrm{Br}_{2}$ Redox Couple in Acetonitrile, Methanol and Mix Media of Acetonitrile-Methanol: An Insight into Redox Behavior of Bromide on Platinum (Pt) and Gold (Au) Electrode. Z. Phys. Chem. 2020, 234, 295-312.

(51) Zari, N.; Mohammedi, H.; Amine, A.; Ennaji, M. M. DNA Hydrolysis and Voltammetric Determination of Guanine and Adenine Using Different Electrodes. Anal. Lett. 2007, 40, 1698-1713.

(52) Ferapontova, E. E. Electrochemistry of Guanine and 8Oxoguanine at Gold Electrodes. Electrochim. Acta 2004, 49, 17511759. 
(53) Link, S.; El-Sayed, M. A. Spectral Properties and Relaxation Dynamics of Surface Plasmon Electronic Oscillations in Gold and Silver Nanodots and Nanorods. J. Phys. Chem. B 1999, 103, 84108426.

(54) Schürmann, R.; Bald, I. Effect of Adsorption Kinetics on Dissociation of DNA-Nucleobases on Gold Nanoparticles under Pulsed Laser Illumination. Phys. Chem. Chem. Phys. 2017, 19, 1079610803.

(55) Yu, L.; Li, N. Binding Strength of Nucleobases and Nucleosides on Silver Nanoparticles Probed by a Colorimetric Method. Langmuir 2016, 32, 5510-5518.

(56) Demers, L. M.; Östblom, M.; Zhang, H.; Jang, N. H.; Liedberg, B.; Mirkin, C. A. Thermal Desorption Behavior and Binding Properties of DNA Bases and Nucleosides on Gold. J. Am. Chem. Soc. 2002, 124, 11248-11249.

(57) Andrews, K. M.; Pearl, T. P. Modification of Ag(111) Surface Electronic Structure via Weak Molecular Adsorption of Adenine Measured with Low Temperature Scanning Tunneling Microscopy and Spectroscopy. J. Chem. Phys. 2010, 132, 214701.

(58) Zhang, X.; Sun, C. Q.; Hirao, H. Guanine Binding to Gold Nanoparticles through Nonbonding Interactions. Phys. Chem. Chem. Phys. 2013, 15, 19284-19292.

(59) Kogikoski, J. S.; Dutta, A.; Bald, I. Spatial Separation of Plasmonic Hot Electron Generation and a Hydrodehalogenation Reaction Center Using a DNA Wire. ChemRxiv, Mar. 1, 2021. DOI: $10.26434 /$ chemrxiv.14114306.v2

(60) Lee, P. C.; Meisel, D. Adsorption and Surface-Enhanced Raman of Dyes on Silver and Gold Sols. J. Phys. Chem. 1982, 86, 3391-3395.

(61) Carnerero, J. M.; Sánchez-Coronilla, A.; Martín, E. I.; JimenezRuiz, A.; Prado-Gotor, R. Quantification of Nucleobases/Gold Nanoparticles Interactions: Energetics of the Interactions through Apparent Binding Constants Determination. Phys. Chem. Chem. Phys. 2017, 19, 22121-22128.

(62) Green, M. A. Self-consistent optical parameters of intrinsic silicon at $300 \mathrm{~K}$ including temperature coefficients. Sol. Energy Mater. Sol. Cells 2008, 92, 1305-1310. 